This pilot suggests further study into the prevalence and management of glucocorticoids in patients with cancer, across all settings, would be beneficial to establish the need for formal guidelines.

\section{IDENTIFYING INAPPROPRIATE PRESCRIBING IN OLDER PEOPLE WITH DEMENTIA: A SYSTEMATIC REVIEW}

Deborah G Hukins, Una Macleod, Jason W Boland. Wolfson Palliative Care Research Centre, Hull York Medical School, University of Hull

\subsection{6/bmjspcare-2018-ASPabstracts.200}

Background Older people with dementia are at risk of adverse drug-related events associated with the prescribing of potentially inappropriate medications.

Aim To describe how tools designed to identify potentially inappropriate prescribing have been used in studies of older people with dementia and to determine the prevalence of potentially inappropriate prescribing in this cohort.

Methods Systematic review with narrative synthesis designed and conducted according to the recommendations set out in Preferred Reporting Items for Systematic Reviews and MetaAnalyses-Protocol (PRISMA-P) and PRISMA statements. Electronic databases, including MEDLINE, EMBASE, PsychINFO, Cochrane Library, Social Science Citation Index, OpenGrey and Grey Literature Report were searched, along with handsearches, for studies using tools/criteria to identify potentially inappropriate prescribing in older people with dementia. Screening, extraction and quality assessment (Hawker score) were performed in duplicate.

Results A total of 3,626 unique records were identified; 26 studies met the inclusion criteria (which included 26,534 participants, of which $21,285(80 \%)$ had dementia or cognitive impairment). The mean participant age in these studies ranged from 72.5 to 86.8. The Hawker score ranged from 22/36 to $36 / 36$, indicating all studies were fair to good quality. The Beer's criteria was the most commonly used tool (15/26 studies). There were variations in how the tools were applied, with 13 out of 15 studies using the Beer's criteria not using the full tool. Eight studies used more than one tool to identify potentially inappropriate prescribing. The prevalence of potentially inappropriate prescribing ranged from $14 \%$ to $74 \%$ in older people with dementia. The most commonly prescribed potentially inappropriate medications were benzodiazepines and anticholinergics.

Conclusions Variations in tool application may partly explain variations in potentially inappropriate prescribing rates across studies. Recommendations include a more standardised tool usage, ensuring the tools comprehensively identify all potentially inappropriate medications and that the tools are kept up to date.

\section{AN OBSERVATIONAL STUDY OF OPIOID-INDUCED CONSTIPATION (OIC)}

C Leach, A Davies. Royal Surrey County Hospital, Guildford

\subsection{6/bmjspcare-2018-ASPabstracts.201}

Background Opioid-induced constipation (OIC) is common, although there is a lack of consensus on diagnostic criteria, and as a result a lack of consistency around the prevalence (with resultant impact on patient care). This study primarily aims to compare different methods of assessing OIC in a heterogeneous group of patients with cancer.

Methods Data was collected on 100 consecutive patients with cancer that were receiving regular opioid analgesics. Constipation was assessed using a simple question ( 'are you constipated'), the EAPC criteria, the so-called 'Camilleri' criteria (for OIC), the Rome IV criteria (for OIC), and assessment by a specialist palliative care professional. The degree of OIC was assessed using the bowel function index (BFI).

Results The median age was 62 (range 37-86); 46\% were female, 54\% were male. Cancer diagnoses were as follows: $34 \%$ gastrointestinal, $13 \%$ gynaecological, $12 \%$ head and neck, 11\% urological, 11\% breast, 10\% lung, 5\% haematological, $2 \%$ skin, $2 \%$ malignancy of unknown origin.

The prevalence of constipation using the different diagnostic criteria was:

- Simple question - 35\% (with 17\% 'unsure')

- EAPC criteria - 29\% (with 6\% 'unsure')

- Camilleri criteria (for OIC) - 63\% (with 6\% 'unsure')

- Rome IV criteria (for OIC) - 71\%

- Assessment by specialist palliative care professional - $68 \%$ had constipation; of which $44 \%$ had OIC.

Of the patients identified as having OIC from the Rome IV criteria, 30\% thought they were constipated, and the mean BFI was 65. In contrast, the mean BFI for patients that did not meet the Rome IV criteria was 32 .

Conclusions The prevalence of OIC depends on the diagnostic criteria employed. It appears that many patients with OIC do not realise that they are in fact constipated (based on objective criteria).

\section{SEIZURE MANAGEMENT IN PATIENTS UNABLE TO TAKE ORAL MEDICATIONS: A MULTI-CENTRE AUDIT}

Alison Cran, Clare Statham, Harriet Bush, Claire Stark-Toller. University Hospital Southampton NHS Foundation Trust

\subsection{6/bmispcare-2018-ASPabstracts.202}

Background Seizures are common in the palliative care population. For patients who are unable to take oral medication, seizure management can become problematic but there is little consensus on best practice, particularly for patients with longer prognoses.

Aim To examine prescribing of non-oral and 'as required' anticonvulsant medication across multiple palliative settings (community/hospice/acute hospital).

Method A regional multi-centre audit of prescribing practice was conducted from October to December 2017. Inclusion criteria: adult patients requiring anticonvulsant medication but unable to take oral preparations. Practice was assessed against regional Palliative Care guidelines. Data was collected retrospectively via a SmartSurvey proforma.

Results Twenty-six patients across six centres were included. When unable to take oral medications, 25 patients (96\%) commenced a continuous subcutaneous infusion (CSCI). Fourteen patients were prescribed levetiracetam (250 mg-3 g/24 hours), seven were prescribed midazolam (15-30 mg/24 hours), two were prescribed sodium valproate $(600 \mathrm{mg} / 24$ hours) and two were prescribed combination levetiracetam (3 g) and midazolam $(30-40 \mathrm{mg})$. One patient had no regular 